\title{
Densidades de plantio e doses de biofertilizante na produção de capim- limão
}

\author{
Arie F Blank ${ }^{1}$; Maria de Fátima Arrigoni-Blank²; Verônica F Amancio ${ }^{1}$; Marcelo da C Mendonça ${ }^{1}$; Luiz G \\ M de Santana Filho ${ }^{1}$
}

${ }^{1}$ UFS DEA, Av. Marechal Rondon s/n, B. Rosa Elze, 49100-000 São Cristóvão-SE; ${ }^{2}$ UFS-Campus Prof. Alberto Carvalho, Av. Vereador Olímpio Grande s/n, 49500-000, Itabaiana-SE; afblank@ufs.br

\section{RESUMO}

O objetivo do trabalho foi avaliar o efeito de densidades de plantio e doses de biofertilizante comercial na produção de capim-limão (Cymbopogon citratus (D.C.) Stapf). O experimento foi implantado em parcelas subdivididas com três repetições, colocando-se nas parcelas as densidades de plantio (33.333, 49.382, 55.555 e 111.111 plantas ha $\left.{ }^{-1}\right)$ e nas subparcelas as doses de biofertilizante $(0 ; 20 ; 40$ e $\left.60 \mathrm{t} \mathrm{ha}^{-1}\right)$. Foram realizadas nove colheitas em intervalos de 42 dias. Avaliou-se a sobrevivência, a altura de planta, o número de perfilhos por touceira, a massa seca por touceira e da parte aérea, o teor e o rendimento de óleo essencial. O teor de óleo essencial não foi influenciado pelas densidades de plantio e doses de biofertilizante. A densidade crescente resultou em regressões lineares crescentes para o rendimento de óleo essencial e a massa seca da parte aérea e em uma regressão linear decrescente para a massa seca por touceira. A dose de biofertilizante resultou em regressões lineares crescentes para o rendimento de óleo essencial e a massa seca por touceira e da parte aérea. A densidade de 111.111 plantas ha $^{-1}(0,30$ x 0,30 m) e a dose de biofertilizante de $60 \mathrm{t} \mathrm{ha}^{-1}$ no primeiro ano de cultivo poderão ser recomendados para produção do capim-limão.

Palavras-chaves: Cymbopogon citratus, planta medicinal, população de plantas, adubação, óleo essencial.

\begin{abstract}
Density and biofertilizer levels for lemon grass production

The aim of this work was to evaluate the plant density and biofertilizer levels on lemon grass (Cymbopogom citratus (D.C.) Stapf). The experiment was installed in split plot design with three replications, having plant density as plots (33,333; 49,382; 55,555 and 111,111 plants ha $\left.^{-1}\right)$ and biofertilizer doses as sub plots (0; 20; 40; $60 \mathrm{t} \mathrm{ha}^{-1}$ year-1 $^{-1}$. Nine cuttings were realized using an interval of 42 days between cuttings. We evaluated survival, plant height, number of tillers per plant, dry weight per plant and of biomass yield, essential oil content and yield. The essential oil content was not influenced by the plant densities and biofertilizer doses. The crescent density resulted in a crescent linear regression for essential oil yield and dry weight of biomass yield and in a declining linear regression for dry weight per plant. The biofertilizer dose resulted in a crescent linear regression for essential oil yield and dry weight per plant and of biomass yield. The plant density of 111.111 plants per hectare $(0.30 \times 0.30 \mathrm{~m})$ and the biofertilizer dose of $60 \mathrm{t} \mathrm{ha}^{-1}$ year $^{-1}$ can be recommended for lemon grass production in the first year.
\end{abstract}

Keywords: Cymbopogon citratus, medicinal plant, plant population, fertilizing, essential oil.

\section{(Recebido para publicação em 29 de agosto de 2006; aceito em 4 de julho de 2007)}

$\mathrm{O}$ gênero Cymbopogon (Poaceae) possui mais de 100 espécies nos países tropicais, inclusive no Brasil (Lorenzi \& Matos, 2002), dentre as quais, aproximadamente 56 são aromáticas. A algumas delas deve-se dar atenção especial pelo seu grande uso na medicina popular e pelo teor de óleo essencial com as mais diferentes finalidades como o uso terapêutico, cosmético e/ou perfumaria. Dentro desse gênero, tem-se o capim-limão (Cymbopogon citratus (DC) Stapf.), originário da Índia, bem conhecido por fornecer óleo essencial rico em citral (84\%) (Ming et al., 1996). No Brasil, é popularmente conhecido por capim-limão, capim-santo, capim-cidreira, capim-cheiroso e capim-de-cheiro. Suas folhas são largamente utilizadas como sedativa, sudorífera, febrífuga, diurética, antipirética e antireumática (Braga, 1976).
O óleo essencial do capim-limão é amplamente utilizado como agente aromatizante na indústria de perfumaria e cosmética, na preparação de sabonetes, colônias e desodorantes, bem como na indústria química para obtenção do citral, que é utilizado como matéria prima para a síntese de ianonas e vitamina A (Guenther, 1992).

O cultivo do capim-limão é extensamente estudado ao nível mundial, em virtude do seu potencial industrial. Entretanto, no Brasil, pouco se sabe a respeito do seu manejo agronômico, principalmente no que se refere a tratos culturais, como densidade de plantio e utilização de biofertilizantes. No caso das espécies medicinais, a densidade de plantio pode favorecer ou não a produtividade em termos de biomassa e também de princípios ativos (Andrade \& Casali, 1999). Na Índia, em plantios comerciais de capim-limão, recomendase que sejam utilizados espaçamentos de $1 \mathrm{~m}$ entre linhas e $0,5 \mathrm{~m}$ entre plantas (20.000 plantas ha-1). Apesar disso, sugere-se a implantação de ensaios em cada local de cultivo para definir a melhor densidade de plantas (Singh et al., 1982).

A matéria orgânica é uma importante fonte de nutrientes para as plantas, microflora e fauna terrestre. No solo, exerce funções importantes como fornecimento de nutrientes e condicionador das propriedades físicas, químicas e biológicas. Além disso, atua diretamente na biologia do solo, constituindo fonte de energia e de nutrientes para os organismos que participam de seu ciclo biológico (Kiehl, 1985).

Em Colombo-PR, testando-se doses de adubação orgânica (0; 1; 2; 4 e 8 kg $\mathrm{m}^{-2}$ de esterco bovino) em Lippia alba (Mill) N.E.Br., verificou-se que o au- 
Tabela 1. Sobrevivência (\%), altura de planta $(\mathrm{cm})$, número de perfilhos por touceira, massa seca por touceira $(\mathrm{g})$ e da parte aérea $(\mathrm{kg}$ ha-1 $)$ de capim-limão nas nove colheitas, em função de diferentes densidades de plantio (survival (\%), plant height (cm), number of tillers per plant, dry mass of plant (g) and of the aerial part ( $\mathrm{kg} \mathrm{ha}^{-1}$ ) of lemon grass on nine harvests, as a function of planting densities). São CristóvãoSE, UFS, 2003.

\begin{tabular}{|c|c|c|c|c|c|}
\hline \multirow{2}{*}{$\begin{array}{l}\text { Densidade } \\
(\text { plantas ha-1) }\end{array}$} & \multirow{2}{*}{$\begin{array}{c}\text { Sobrevivência } \\
(\%)\end{array}$} & \multirow{2}{*}{$\begin{array}{l}\text { Altura de planta } \\
(\mathrm{cm})\end{array}$} & \multirow{2}{*}{$\begin{array}{l}\mathbf{N}^{\circ} \text { perfilhos } / \\
\text { touceira }\end{array}$} & \multicolumn{2}{|c|}{ Massa seca } \\
\hline & & & & Touceira $(g)$ & Parte aérea $\left(\mathrm{kg} \mathrm{ha}^{-1}\right)$ \\
\hline \multicolumn{6}{|c|}{ Primeira colheita $(22 / 03 / 2002)$} \\
\hline 33.333 & 96,67 & 60,65 & 4,89 & 11,57 & 385,57 \\
\hline 49.382 & 97,50 & 60,58 & 4,79 & 11,37 & 561,61 \\
\hline 55.555 & 98,33 & 64,61 & 4,81 & 13,08 & 726,38 \\
\hline 111.111 & 95,83 & 64,40 & 4,83 & 11,29 & 1254,60 \\
\hline C.V. (A) $-\%$ & 6,59 & 7,18 & 40,36 & 25,98 & 29,53 \\
\hline C.V. (B) - \% & 5,82 & 8,93 & 24,81 & 33,86 & 35,40 \\
\hline Equação $(\hat{y}=)$ & ns & ns & ns & ns & $\begin{array}{l}46,08+0,01^{* *} x \\
R^{2}=0,9847\end{array}$ \\
\hline \multicolumn{6}{|c|}{ Segunda colheita $(03 / 05 / 2002)$} \\
\hline 33.333 & 94,17 & 59,78 & 7,47 & 12,56 & 418,62 \\
\hline 49.382 & 95,83 & 64,47 & 8,64 & 14,84 & 732,66 \\
\hline 55.555 & 95,83 & 67,31 & 10,00 & 14,93 & 829,64 \\
\hline 111.111 & 90,00 & 70,72 & 7,81 & 12,41 & 1379,25 \\
\hline C.V. $(A)-\%$ & 9,04 & 11,59 & 30,64 & 41,48 & 56,95 \\
\hline C.V. (B) $-\%$ & 9,17 & 9,36 & 25,57 & 31,74 & 26,17 \\
\hline Equação $(\hat{y}=)$ & ns & $57,91+10^{-4 *} \times R^{2}=0,8053$ & $\begin{array}{l}0,86+3 \cdot 10^{-4} x-10-7^{*} x^{2} \\
R^{2}=0,8865\end{array}$ & ns & $\begin{array}{l}114,12+0,01^{* *} \mathrm{x} \\
\mathrm{R}^{2}=0,9700\end{array}$ \\
\hline \multicolumn{6}{|c|}{ Terceira colheita $(14 / 06 / 2002)$} \\
\hline 33.333 & 94,17 & 62,89 & 15,56 & 14,33 & 477,67 \\
\hline 49.382 & 95,83 & 68,58 & 16,67 & 13,62 & 672,82 \\
\hline 55.555 & 94,17 & 71,33 & 15,69 & 15,02 & 834,62 \\
\hline 111.111 & 86,67 & 72,92 & 10,64 & 11,13 & 1236,70 \\
\hline C.V. $(A)-\%$ & 10,95 & 5,095 & 23,50 & 27,38 & 37,67 \\
\hline C.V. (B) $-\%$ & 11,49 & 6,197 & 32,26 & 37,32 & 30,14 \\
\hline Equação $(\hat{y}=)$ & ns & $\begin{array}{l}42,67+7 \cdot 10^{-4} x-10-7^{* *} x^{2} \\
\mathrm{R}^{2}=0,9927\end{array}$ & $\begin{array}{l}19,27-7 \cdot 10^{-4 * *} x \\
\mathrm{R}^{2}=0,8589\end{array}$ & $\begin{array}{l}16,28-4 \cdot 10^{-5 *} x \\
R^{2}=0,7749\end{array}$ & $\begin{array}{l}223,36+0,009^{* *} x \\
\mathrm{R}^{2}=0,9599\end{array}$ \\
\hline \multicolumn{6}{|c|}{ Quarta colheita (26/07/2002) } \\
\hline 33.333 & 90,00 & 62,14 & 8,92 & 20,34 & 678,05 \\
\hline 49.382 & 90,00 & 70,03 & 10,28 & 19,66 & 970,82 \\
\hline 55.555 & 90,00 & 71,83 & 11,64 & 26,07 & 1448,13 \\
\hline 111.111 & 85,00 & 71,17 & 6,94 & 15,38 & 1709,31 \\
\hline C.V. $(A)-\%$ & 15,03 & 6,815 & 30,18 & 28,03 & 40,25 \\
\hline C.V. (B) $-\%$ & 14,09 & 7,855 & 29,45 & 41,73 & 35,10 \\
\hline Equação $(\hat{y}=)$ & ns & $\begin{array}{l}36,62+0,001 x-10^{-7 * *} x^{2} \\
\mathrm{R}^{2}=0,9990\end{array}$ & $\begin{array}{l}0,75+3 \cdot 10-4 x-10^{-7 *} x^{2} \\
R 2=0,9629\end{array}$ & $\begin{array}{l}6,23+5 \cdot 10-4 x-10^{-7 *} x^{2} \\
R^{2}=0,7032\end{array}$ & $\begin{array}{l}452,38+0,01^{* *} x \\
\mathrm{R}^{2}=0,7677\end{array}$ \\
\hline \multicolumn{6}{|c|}{ Quinta colheita (06/09/2002) } \\
\hline 33.333 & 90,00 & 64,50 & 11,42 & 29,61 & 986,84 \\
\hline 49.382 & 88,33 & 68,65 & 12,17 & 26,87 & 1326,67 \\
\hline 55.555 & 90,00 & 72,75 & 12,55 & 30,19 & 1677,80 \\
\hline 111.111 & 85,00 & 68,47 & 8,50 & 16,53 & 1836,80 \\
\hline C.V. $(A)-\%$ & 10,33 & 6,53 & 11,02 & 20,19 & 24,20 \\
\hline C.V. (B) $-\%$ & 15,39 & 8,88 & 30,21 & 41,66 & 33,60 \\
\hline Equação $(\hat{y}=)$ & ns & $\begin{array}{l}43,74+8 \cdot 10^{-4} x-107^{* *} x^{2} \\
R^{2}=0,9021\end{array}$ & $\begin{array}{l}6,98+2 \cdot 10^{-4} x-10^{-7 * *} x^{2} \\
R^{2}=0,9969\end{array}$ & $\begin{array}{l}36,80-2 \cdot 10^{-4 * *} x \\
\mathrm{R}^{2}=0,8854\end{array}$ & $\begin{array}{l}869,41+0,009^{* *} x \\
R^{2}=0,7078\end{array}$ \\
\hline \multicolumn{6}{|c|}{ Sexta colheita $(18 / 10 / 2002)$} \\
\hline 33.333 & 85,83 & 65,19 & 14,17 & 18,59 & 619,67 \\
\hline 49.382 & 87,50 & 63,38 & 14,89 & 18,05 & 891,53 \\
\hline 55.555 & 88,33 & 68,03 & 13,22 & 16,51 & 917,18 \\
\hline 111.111 & 81,67 & 67,27 & 12,25 & 12,68 & 1409,12 \\
\hline C.V. (A) - \% & 15,74 & 3,63 & 19,67 & 17,66 & 24,04 \\
\hline C.V. (B) $-\%$ & 15,68 & 7,80 & 22,95 & 30,57 & 30,64 \\
\hline Equação $(\hat{y}=)$ & ns & ns & ns & $\begin{array}{l}21,31-8 \cdot 10^{-5 * *} \mathrm{x} \\
\mathrm{R}^{2}=0,9724\end{array}$ & $\begin{array}{l}361,09+0,01^{* *} x \\
\mathrm{R}^{2}=0,9759\end{array}$ \\
\hline
\end{tabular}


Tabela 1. Continuação

\begin{tabular}{|c|c|c|c|c|c|}
\hline \multirow{2}{*}{$\begin{array}{l}\text { Densidade } \\
\text { (plantas ha-1) }\end{array}$} & \multirow{2}{*}{$\begin{array}{c}\text { Sobrevivência } \\
(\%)\end{array}$} & \multirow{2}{*}{$\begin{array}{l}\text { Altura de planta } \\
(\mathrm{cm})\end{array}$} & \multirow{2}{*}{$\begin{array}{l}\mathrm{N}^{\circ} \text { perfilhos/ } \\
\text { touceira }\end{array}$} & \multicolumn{2}{|c|}{ Massa seca } \\
\hline & & & & Touceira $(\mathbf{g})$ & Parte aérea $\left(\mathrm{kg} \mathrm{ha}^{-1}\right)$ \\
\hline \multicolumn{6}{|c|}{ Sétima colheita $(29 / 11 / 2002)$} \\
\hline 33.333 & 78,33 & 66,58 & 13,86 & 16,63 & 474,89 \\
\hline 49.382 & 88,33 & 71,56 & 15,97 & 19,00 & 804,18 \\
\hline 55.555 & 83,33 & 70,27 & 16,11 & 19,11 & 909,53 \\
\hline 111.111 & 80,00 & 70,69 & 15,28 & 15,03 & 1430,80 \\
\hline C.V. (A) - \% & 21,78 & 6,59 & 17,25 & 47,46 & 47,41 \\
\hline C.V. (B) - \% & 20,02 & 10,31 & 24,56 & 47,97 & 42,75 \\
\hline Equação (̂ิ= ) & ns & ns & ns & ns & $\begin{array}{c}189,60+0,01^{* *} x \\
R^{2}=0,9583\end{array}$ \\
\hline \multicolumn{6}{|c|}{ Oitava colheita $(10 / 01 / 2003)$} \\
\hline 33.333 & 77,50 & 68,00 & 16,14 & 14,80 & 493,43 \\
\hline 49.382 & 84,17 & 76,49 & 18,25 & 23,42 & 1156,82 \\
\hline 55.555 & 81,67 & 73,80 & 16,36 & 18,80 & 1044,55 \\
\hline 111.111 & 75,83 & 75,44 & 14,03 & 13,32 & 1479,73 \\
\hline C.V. (A) - \% & 23,28 & 12,12 & 22,94 & 52,27 & 41,80 \\
\hline C.V. (B) - \% & 19,34 & 14,56 & 19,11 & 46,60 & 41,14 \\
\hline Equação (̂̂y= ) & ns & ns & ns & ns & $\begin{array}{c}394,46+0,01^{*} x \\
R^{2}=0,7362\end{array}$ \\
\hline \multicolumn{6}{|c|}{ Nona colheita $(21 / 02 / 2003)$} \\
\hline 33.333 & 78,33 & 68,18 & 29,38 & 9,59 & 319,93 \\
\hline 49.382 & 85,83 & 71,53 & 22,02 & 15,92 & 786,58 \\
\hline 55.555 & 81,67 & 71,53 & 30,61 & 13,61 & 756,04 \\
\hline 111.111 & 75,83 & 71,72 & 18,64 & 9,38 & 1042,68 \\
\hline C.V. (A) - \% & 19,33 & 12,39 & 16,51 & 92,19 & 79,64 \\
\hline C.V. (B) - \% & 21,19 & 13,45 & 22,31 & 88,62 & 76,83 \\
\hline Equação (̂ิ= ) & ns & ns & $\begin{array}{c}33,10-10-4^{* *} x \\
R 2=0,6569\end{array}$ & ns & $\begin{array}{c}250,00+0,008^{*} x \\
R 2=0,7430\end{array}$ \\
\hline
\end{tabular}

mento nas doses de esterco bovino resultou em maiores rendimentos de biomassa, porém, em decréscimo no teor de óleo essencial (Ming, 1998).

Em solo sob uso intensivo recomenda-se, para a cultura do capim-limão, a aplicação de $62 \mathrm{~kg} \mathrm{ha}^{-1}$ de $\mathrm{P}_{2} \mathrm{O}_{5}$ e $50 \mathrm{~kg}$ $\mathrm{ha}^{-1}$ de $\mathrm{K}_{2} \mathrm{O}$ no momento do plantio, além de $125 \mathrm{~kg} \mathrm{ha}^{-1}$ de $\mathrm{N}$ distribuídos durante o ciclo da planta. A aplicação foliar de uréia a $1 \%$ foi bastante eficiente e, em caso de necessidade, recomendase a aplicação de pequena dose de fósforo e potássio em cobertura. Após cada colheita, aplicam-se as mesmas quantidades de fertilizantes que foram aplicadas no plantio (Singh et al., 1982).

Com doses de $185 \mathrm{~kg} \mathrm{ha}^{-1}$ de N, Bhan et al. (1999) obtiveram em seus experimentos com capim-limão, conduzidos na Índia, rendimento de óleo superior a 349 kg ha-1. Já Khode et al. (1999) relacionaram a densidade de plantio com diferentes doses de $\mathrm{N}$ e encontraram melhores resultados de biomassa seca sob densidade de 55.555 plantas ha-1 e doses de $\mathrm{N}$ superiores a $100 \mathrm{~kg} \mathrm{ha}^{-1}$, utilizando-se cobertura morta para proteção do solo.

A produção de óleo essencial do capim-limão em três estações diferentes, com quatro intervalos de um mês em cada estação, foi determinada por Leal et al. (2001) em Sobral-CE. O teor de óleo essencial alcançou seu maior nível em outubro de 1997 (acima de 1,5 ml $100 \mathrm{~g}^{-1}$ de massa seca), diminuiu significativamente em fevereiro de 1998 (0,9 $\mathrm{ml} 100 \mathrm{~g}^{-1}$ de massa seca), culminando com maior redução em junho de 1998 (0,4 $\mathrm{ml} 100 \mathrm{~g}^{-1}$ de massa seca).

O objetivo deste trabalho foi avaliar o efeito de diferentes densidade de plantio e doses de biofertilizante comercial na produção do capim-limão.

\section{MATERIAL E MÉTODOS}

O trabalho foi em Santana do São Francisco, Sergipe. O solo é do tipo
Argissolo Vermelho Amarelo com as características: $\mathrm{pH}$ (em água) 5,4; P, Zn, Fe, Mn, Cu, B e S (mg dm${ }^{-3}$ ): 17,9; 1,0; 115,0; 1,2; 0,2; 0,3 e 6,6; K, Ca, Mg, $\mathrm{Al}^{+3}, \mathrm{H}+$ $\mathrm{Al}, \mathrm{SB}$, t e T (cmol $\left.\mathrm{dm}^{-3}\right): 0,05 ; 0,7 ; 0,2$; 0,2; 1,9; 1,0; 1,2 e 2,9; V e m (\%): 33,6 e 17 e $\mathrm{MO}$ - 11,0 $\mathrm{g} \mathrm{dm}^{-3}$. Foi feita a correção da acidez utilizando-se calcário dolomítico para ajustar o pH para 6,0.

Utilizaram-se mudas de capim-limão, provenientes de matrizes existentes no Horto de Plantas Medicinais da UFS. O experimento foi implantado em parcelas subdivididas, usando-se nas parcelas as densidades de plantio (33.333; 49.382; 55.555 e 111.111 plantas ha ${ }^{-1}$ ) e nas subparcelas, as doses de biofertilizante comercial Vitasolo â (0; 20; 40 e 60 tha $\left.^{-1}\right)$, com três repetições. Cada subparcela foi constituída por quatro fileiras de sete plantas. $\mathrm{O}$ biofertilizante Vitasoloa apresentou as características: $\mathrm{pH}$ (em água): 8,3; $\mathrm{MO}$ - 173,0 $\mathrm{g} \mathrm{dm}^{-3}$; P, Zn, Cu, Fee Mn (mg dm ${ }^{-3}$ ): 993,7; 2,78; 2,86; 26,55 e 50,43; K, Ca + Mg e S$\mathrm{SO}_{4}^{-2}\left(\mathrm{cmol}_{\mathrm{c}} \mathrm{dm}^{-3}\right): 15,35 ; 11,25$ e 33,32 . 
Tabela 2. Sobrevivência (\%), altura de planta (cm), número de perfilhos por touceira, massa seca por touceira (g) e da parte aérea (kg ha-1) de capim-limão nas nove colheitas, em função de doses de biofertilizante Vitasoloâ (survival (\%), plant height (cm), number of tillers per plant, dry mass of plant (g) and of the aerial part $\left(\mathrm{kg} \mathrm{ha}^{-1}\right)$ of lemon grass on nine harvests, as a function of the biofertilizer Vitasolo $\left.{ }^{\hat{a}}\right)$. São Cristóvão-SE, UFS, 2003.

\begin{tabular}{|c|c|c|c|c|c|}
\hline \multirow{2}{*}{$\begin{array}{l}\text { Dose de } \\
\text { biofertilizante } \\
\left(\mathrm{kg} \mathrm{ha}^{-1}\right)\end{array}$} & \multirow{2}{*}{$\begin{array}{c}\text { Sobrevivência } \\
(\%)\end{array}$} & \multirow{2}{*}{$\begin{array}{l}\text { Altura de planta } \\
\qquad(\mathrm{cm})\end{array}$} & \multirow{2}{*}{$\begin{array}{c}N^{\circ} \text { perfilhos } / \\
\text { touceira }\end{array}$} & \multicolumn{2}{|c|}{ Massa seca } \\
\hline & & & & Touceira (g) & Parte aérea $\left(\mathrm{kg} \mathrm{ha}^{-1}\right)$ \\
\hline \multicolumn{6}{|c|}{ Primeira colheita $(22 / 03 / 2002)$} \\
\hline 0 & 97,50 & 62,21 & 4,44 & 10,05 & 636,66 \\
\hline 20 & 94,17 & 59,60 & 4,59 & 10,75 & 662,17 \\
\hline 40 & 99,17 & 65,07 & 5,23 & 13,40 & 802,72 \\
\hline 60 & 97,50 & 63,36 & 5,06 & 13,10 & 826,60 \\
\hline Equação (ŷy= ) & ns & ns & ns & $\begin{array}{c}10,06+0,06^{*} x \\
R^{2}=0,8278\end{array}$ & $\begin{array}{c}625,48+3,55^{*} x \\
R^{2}=0,9038\end{array}$ \\
\hline \multicolumn{6}{|c|}{ Segunda colheita $(03 / 05 / 2002)$} \\
\hline 0 & 95,00 & 61,44 & 8,47 & 10,88 & 692,32 \\
\hline 20 & 90,00 & 64,06 & 7,64 & 13,11 & 802,36 \\
\hline 40 & 96,67 & 68,78 & 9,08 & 15,40 & 918,71 \\
\hline 60 & 94,17 & 68,00 & 8,72 & 15,36 & 946,79 \\
\hline Equação $(\hat{y}=)$ & ns & $\begin{array}{c}61,91+0,12^{* *} x \\
R^{2}=0,8375\end{array}$ & ns & $\begin{array}{c}11,33+0,08^{* *} x \\
R^{2}=0,8869\end{array}$ & $\begin{array}{c}708,08+4,40^{* *} x \\
R^{2}=0,9479\end{array}$ \\
\hline \multicolumn{6}{|c|}{ Terceira colheita $(14 / 06 / 2002)$} \\
\hline 0 & 90,00 & 66,11 & 11,42 & 10,64 & 682,36 \\
\hline 20 & 90,00 & 67,64 & 13,42 & 12,62 & 764,07 \\
\hline 40 & 97,50 & 71,72 & 17,39 & 15,56 & 882,63 \\
\hline 60 & 94,17 & 70,25 & 16,33 & 15,28 & 892,74 \\
\hline Equação $(\hat{y}=)$ & ns & $\begin{array}{c}66,46+0,08^{* *} x \\
R^{2}=0,7108\end{array}$ & $11,83+0,09^{* *} x$ & $R^{2}=0,7856$ & $\begin{array}{c}11,00+0,08^{*} x \\
R^{2}=0,8686\end{array}$ \\
\hline \multicolumn{6}{|c|}{ Quarta colheita $(26 / 07 / 2002)$} \\
\hline 0 & 86,67 & 65,36 & 7,64 & 14,80 & 949,79 \\
\hline 20 & 85,00 & 67,33 & 9,47 & 18,52 & 1069,31 \\
\hline 40 & 92,50 & 71,11 & 10,81 & 25,39 & 1466,87 \\
\hline 60 & 90,00 & 71,36 & 9,86 & 22,74 & 1320,33 \\
\hline Equação $(\hat{y}=)$ & ns & $\begin{array}{c}65,52+0,11^{* *} x \\
R^{2}=0,9163\end{array}$ & $\begin{array}{c}8,24+0,04^{*} x \\
R^{2}=0,6048\end{array}$ & $\begin{array}{c}15,76+0,15^{* *} x \\
R^{2}=0,7209\end{array}$ & $\begin{array}{c}975,20+7,55^{*} x \\
R^{2}=0,6886\end{array}$ \\
\hline \multicolumn{6}{|c|}{ Quinta colheita (06/09/2002) } \\
\hline 0 & 86,67 & 65,75 & 9,30 & 19,19 & 1172,53 \\
\hline 20 & 82,50 & 67,77 & 11,08 & 22,74 & 1266,23 \\
\hline 40 & 95,83 & 70,40 & 12,00 & 30,55 & 1660,07 \\
\hline 60 & 88,33 & 70,44 & 12,25 & 30,72 & 1728,60 \\
\hline Equação $(\hat{y}=)$ & ns & $\begin{array}{c}66,09+0,08^{*} x \\
R^{2}=0,9034\end{array}$ & $\begin{array}{c}9,70+0,05^{*} x \\
R^{2}=0,8902\end{array}$ & $\begin{array}{c}19,44+0,21^{* *} x \\
R^{2}=0,9005\end{array}$ & $\begin{array}{c}1147,56+10,31^{* *} x \\
R^{2}=0,9151\end{array}$ \\
\hline \multicolumn{6}{|c|}{ Sexta colheita $(18 / 10 / 2002)$} \\
\hline 0 & 84,17 & 63,40 & 10,80 & 11,59 & 748,95 \\
\hline 20 & 81,67 & 64,69 & 12,56 & 15,20 & 858,22 \\
\hline 40 & 93,33 & 68,80 & 15,53 & 19,53 & 1123,64 \\
\hline 60 & 84,17 & 66,97 & 15,64 & 19,52 & 1106,68 \\
\hline Equação $(\hat{y}=)$ & ns & $\begin{array}{c}63,75+0,07^{*} x \\
R^{2}=0,6360\end{array}$ & $\begin{array}{c}11,01+0,09^{* *} x \\
R^{2}=0,9103\end{array}$ & $\begin{array}{c}12,24+0,14^{* *} \mathrm{x} \\
\mathrm{R}^{2}=0,8966\end{array}$ & $\begin{array}{c}758,58+6,69^{* *} x \\
R^{2}=0,8682\end{array}$ \\
\hline \multicolumn{6}{|c|}{ Sétima colheita $(29 / 11 / 2002)$} \\
\hline 0 & 77,50 & 67,03 & 13,17 & 11,34 & 715,55 \\
\hline 20 & 80,83 & 67,67 & 14,83 & 13,54 & 839,69 \\
\hline 40 & 90,83 & 71,14 & 16,17 & 16,30 & 964,81 \\
\hline 60 & 80,83 & 73,28 & 17,06 & 18,60 & 1099,35 \\
\hline Equação $(\hat{y}=)$ & ns & $\begin{array}{c}66,44+0,11^{*} x \\
R^{2}=0,9452\end{array}$ & $\begin{array}{c}13,36+0,06^{*} x \\
R^{2}=0,9824\end{array}$ & $\begin{array}{c}11,27+0,12^{*} x \\
R^{2}=0,9982\end{array}$ & $\begin{array}{c}713,37+6,38^{*} x \\
R^{2}=0,9996\end{array}$ \\
\hline
\end{tabular}


Tabela 2. Continuação

\begin{tabular}{|c|c|c|c|c|c|}
\hline \multirow{2}{*}{$\begin{array}{l}\text { Dose de } \\
\text { biofertilizante } \\
\left(\mathrm{kg} \mathrm{ha}^{-1}\right)\end{array}$} & \multirow{2}{*}{$\begin{array}{c}\text { Sobrevivência } \\
\text { (\%) }\end{array}$} & \multirow{2}{*}{$\begin{array}{l}\text { Altura de planta } \\
\qquad(\mathrm{cm})\end{array}$} & \multirow{2}{*}{$\begin{array}{c}N^{\circ} \text { perfilhos/ } \\
\text { touceira }\end{array}$} & \multicolumn{2}{|c|}{ Massa seca } \\
\hline & & & & Touceira (g) & Parte aérea $\left(\mathrm{kg} \mathrm{ha}^{-1}\right)$ \\
\hline \multicolumn{6}{|c|}{ Oitava colheita $(10 / 01 / 2003)$} \\
\hline 0 & 77,50 & 72,67 & 13,33 & 12,69 & 723,06 \\
\hline 20 & 75,83 & 72,17 & 15,19 & 14,98 & 925,04 \\
\hline 40 & 87,50 & 75,28 & 17,39 & 18,48 & 1099,57 \\
\hline 60 & 78,33 & 73,64 & 18,86 & 24,21 & 1426,85 \\
\hline Equação $(\hat{y}=)$ & ns & ns & $\begin{array}{c}13,38+0,09^{* *} x \\
R^{2}=0,9947\end{array}$ & $\begin{array}{c}11,87+0,19^{*} x \\
R^{2}=0,9607\end{array}$ & $\begin{array}{c}700,74+11,43^{*} x \\
R^{2}=0,9792\end{array}$ \\
\hline \multicolumn{6}{|c|}{ Nona colheita $(21 / 02 / 2003)$} \\
\hline 0 & 75,83 & 70,29 & 21,70 & 9,54 & 559,14 \\
\hline 20 & 78,33 & 68,83 & 23,78 & 10,93 & 671,97 \\
\hline 40 & 90,00 & 76,14 & 27,53 & 13,72 & 848,93 \\
\hline 60 & 77,50 & 67,70 & 27,67 & 14,35 & 825,20 \\
\hline Equação $(\hat{y}=)$ & ns & ns & $\begin{array}{c}21,92+0,11^{* *} x \\
R^{2}=0,9094\end{array}$ & $\begin{array}{c}9,54+0,09^{*} x \\
R^{2}=0,9504\end{array}$ & $\begin{array}{c}580,04+4,88^{*} x \\
R^{2}=0,8534\end{array}$ \\
\hline
\end{tabular}

O plantio foi realizado em 12/12/2001 e os tratamentos de adubação com biofertilizante Vitasoloa feitos no plantio e em cobertura após cada colheita ímpar das lâminas foliares. O sistema de irrigação adotado foi por aspersão e foi feita quando necessária. Realizaram-se capinas manuais para manter a cultura no limpo.

Realizaram-se nove colheitas das lâminas foliares, sendo a primeira aos 72 dias após o plantio e a partir daí, a intervalos de 42 dias. Foram avaliadas as variáveis nas colheitas: sobrevivência das plantas, altura de planta, número de perfilhos por touceira, massa seca por touceira e da parte aérea, teor e rendimento de óleo essencial, este último a partir da sexta colheita. O material colhido foi seco em estufa de circulação de ar forçada à temperatura de $40+1^{\circ} \mathrm{C}$, até massa constante. $\mathrm{O}$ óleo essencial das folhas secas foi extraído pelo método de hidrodestilação em aparelho Clevenger, por três horas, no Laboratório de Fitotecnia, da UFS, expressandose os resultados de teor em $\mathrm{ml} 100 \mathrm{~g}^{-1}$ de massa seca da parte aérea.

Os dados foram submetidos à análise de variância e aplicou-se a regressão polinomial às variáveis avaliadas de cada colheita. Utilizou-se o programa SISVAR ${ }^{\circledR}$ para as análises estatísticas.

\section{RESULTADOS E DISCUSSÃO}

Na análise de variância não se observou interação significativa entre

Tabela 3. Massa seca total por touceira e da parte aérea e rendimento de óleo essencial de capim-limão durante nove colheitas (total dry weight of plants and of aerial part and yield of essential oil of lemon grass, during nine harvests). São Cristóvão-SE, UFS, 2003.

\begin{tabular}{|c|c|c|c|}
\hline \multirow{2}{*}{$\begin{array}{l}\text { Densidade } \\
\text { (plantas ha } \mathbf{h}^{-1} \text { ) }\end{array}$} & \multicolumn{2}{|c|}{ Massa seca } & \multirow{2}{*}{$\begin{array}{l}\text { Rendimento de óleo } \\
\text { essencial }\left(\mathrm{l} \mathrm{ha}{ }^{-1}\right)^{1 /}\end{array}$} \\
\hline & Touceira $(\mathbf{g})$ & $\begin{array}{c}\text { Parte aérea } \\
\left(\mathrm{kg} \mathrm{ha}^{-1}\right)\end{array}$ & \\
\hline 33.333 & 148,58 & $4.952,81$ & 37,32 \\
\hline 49.382 & 163,66 & $8.082,14$ & 66,87 \\
\hline 55.555 & 168,03 & $9.334,96$ & 61,23 \\
\hline 111.111 & 118,08 & $13.120,39$ & 101,27 \\
\hline Equação ( $\hat{y}=)$ & $\begin{array}{c}182,54-0,0005^{*} x \\
R^{2}=0,6262\end{array}$ & $\begin{array}{c}2.918,01+0,096^{* *} x \\
R^{2}=0,9147\end{array}$ & $\begin{array}{c}19,95+0,0007^{* *} x \\
R^{2}=0,9233\end{array}$ \\
\hline \multicolumn{4}{|c|}{$\begin{array}{l}\text { Dose de } \\
\text { biofertilizante }\left(t \mathrm{ha}^{-1}\right)\end{array}$} \\
\hline 0 & 113,48 & $7.073,39$ & 52,31 \\
\hline 20 & 135,21 & $8.034,62$ & 58,52 \\
\hline 40 & 171,76 & $9.971,06$ & 70,22 \\
\hline 60 & 177,89 & $10.411,23$ & 80,63 \\
\hline Equação ( $\hat{y}=$ ) & $\begin{array}{c}115,12+1,15^{\star *} x \\
R^{2}=0,9418\end{array}$ & $\begin{array}{c}7.080,09+59,75^{* *} x \\
R^{2}=0,9503\end{array}$ & $\begin{array}{c}49,92+0,56^{* *} x \\
R^{2}=0,9670\end{array}$ \\
\hline C.V. (A) $-\%$ & 27,33 & 28,53 & 45,37 \\
\hline C.V. (B) $-\%$ & 35,75 & 29,37 & 41,45 \\
\hline
\end{tabular}

${ }^{1 /}$ Somatório das quatro últimas colheitas ( ${ }^{1 /}$ total of four harvests).

densidades de plantio e doses de biofertilizante para as variáveis avaliadas.

A sobrevivência das plantas não foi influenciada pela densidade de plantio nem pelas doses de biofertilizante aplicadas, mas houve diminuição até a nona colheita (Tabelas 1 e 2). Estes resultados demonstram que as plantas de capim limão se esgotam pelos cortes a cada 42 dias.

A altura de planta foi influenciada pela densidade de plantio entre a segun- da e quinta colheitas (Tabela 1), sendo que após esta colheita notou-se fechamento total do ensaio. Observou-se resposta linear na segunda colheita e quadrática na terceira, quarta e quinta colheitas. Isto demonstra que no início do cultivo não houve competição entre as touceiras e a partir da terceira, a competição limitou o crescimento em altura.

Observou-se resposta linear em função da densidade de plantio para as características rendimento de biomassa e 


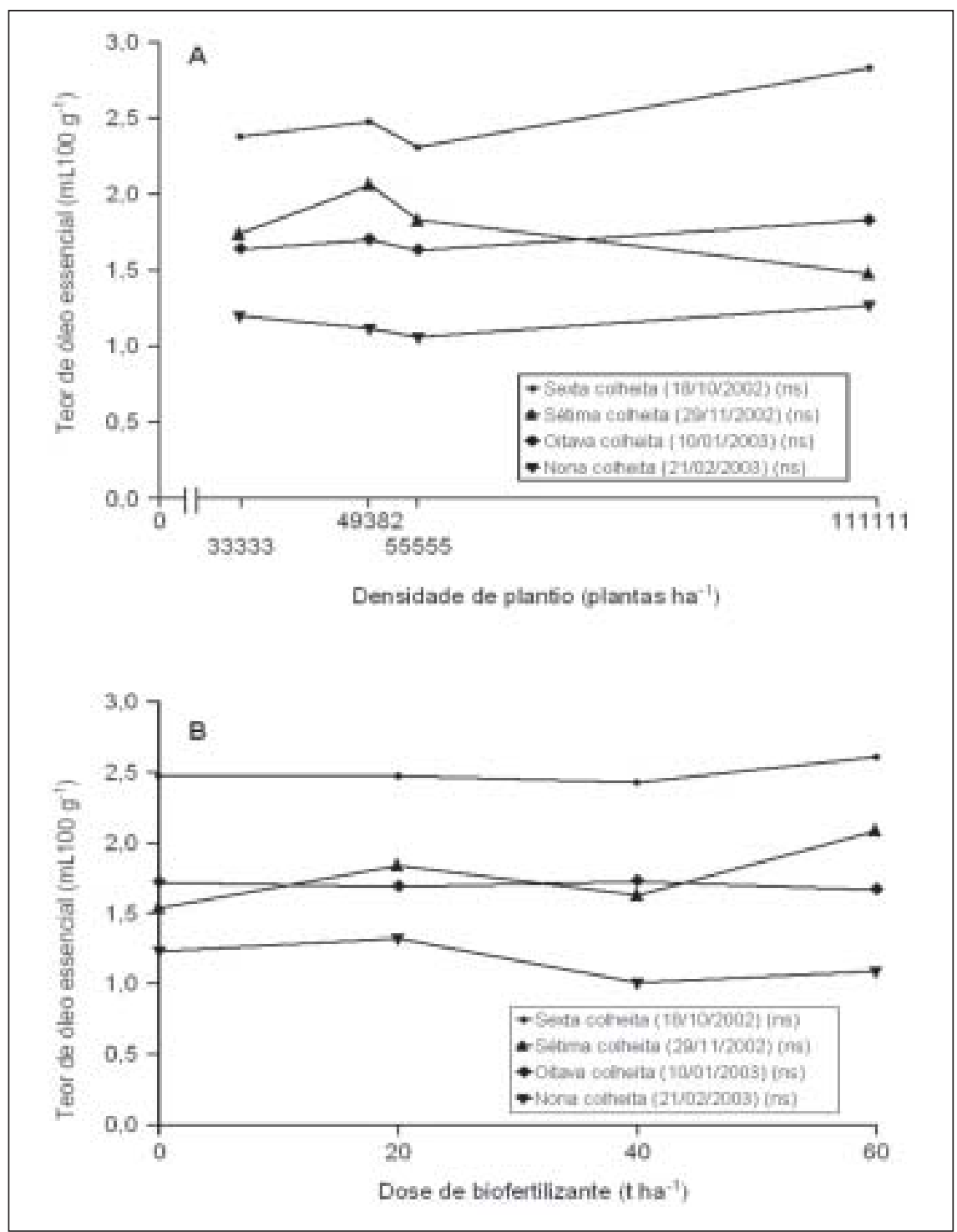

Figura 1. Teor médio ( $\mathrm{ml} 100 \mathrm{~g}^{-1}$ ) de óleo essencial de capim-limão entre a sexta e nona colheita, em função de diferentes densidades de plantio (A) e doses de biofertilizantes (B) (mean value $\left(\mathrm{ml} 100 \mathrm{~g}^{-1}\right)$ of essential oil of lemon grass on the $6^{\text {th }}$ to $9^{\text {th }}$ harvest, depending on planting densities (A) and biofertilizer doses (B)). São Cristóvão-SE, UFS, 2003.

óleo essencial (Tabela 1 e Figura 2A). Este resultado é conseqüência direta da maior densidade de plantas, já que a massa seca por touceira não foi influenciada nas colheitas $1,2,7$ e 8 e diminuiu com a densidade de plantio de 111.111 plantas ha ${ }^{-1}$ (Tabela 1$)$. Resposta linear para doses de biofertilizante Vitasolo ${ }^{a}$ foi notada em todas as colheitas para rendimento de massa seca e óleo essencial (Tabela 2 e Figura 2B).

$\mathrm{O}$ teor de óleo essencial não foi influenciado pelas densidades de plantio e doses de biofertilizante utilizadas ( $\mathrm{Fi}$ gura 1). Silva et al. (2003), trabalhando touceiras causado pelo aumento da densidade de plantio é insuficiente para provocar uma redução da produção de capim-limão.

A densidade de 111.111 plantas ha${ }^{1}$ (espaçamento 0,30 x 0,30 m) apresentou as melhores médias na maioria das características avaliadas, principalmente para rendimento de massa seca e rendimento de óleo essencial. Contudo Khode et al. (1999) obtiveram resultado diferente ao relacionarem a densidade de plantio com doses de $\mathrm{N}$, encontrando melhores resultados em relação à massa seca na densidade 55.555 plantas ha ${ }^{-1}$. Já Dhar et al. (1996), chegaram a uma conclusão semelhante. Avaliaram cinco espaçamentos $(0,30 \times 0,30 \mathrm{~m}, 0,35 \mathrm{x}$ $0,35 \mathrm{~m}, 0,40 \times 0,40 \mathrm{~m}$ e $0,50 \times 0,50$ $\mathrm{m})$ e, embora o rendimento de óleo essencial e massa fresca tenham sido maiores sob o espaçamento $0,50 \times 0,50$ $\mathrm{m}$, o espaçamento $0,30 \times 0,30 \mathrm{~m}$ (111.111 plantas ha-1) foi indicado por ter uma maior projeção de rendimento por area.

A dose de biofertilizante de $60 \mathrm{t}$ $\mathrm{ha}^{-1}$ proporcionou as maiores médias em todas as variáveis avaliadas e houve diferenças significativas entre os tratamentos (Tabela 3). Oliveira et al. (2002), avaliando o efeito da adubação mineral e orgânica sobre a produção de massa e óleo essencial de capim-limão, concluíram que somente com a fertilização orgânica é possível fazer vários cortes e obter alta produtividade, o que confirma os resultados obtidos.

A densidade de 111.111 plantas ha ${ }^{-1}$ $(0,30 \times 0,30 \mathrm{~m})$ e a dose de biofertilizante de $60 \mathrm{t} \mathrm{ha}^{-1}$ no primeiro ano de plantio poderão ser recomendados para produção de capim-limão.

\section{AGRADECIMENTOS} para as densidades de plantio, demonstrando que a competição é maior quando se usa a maior densidade. Para o rendimento de massa seca da parte aérea e de óleo essencial observou-se uma resposta linear crescente para a densidade de plantio (Tabela 3). Estes resultados demonstram que a diminuição das 


\section{LITERATURA CITADA}

ANDRADE FMC; CASALI VWD. 1999. Plantas medicinais e aromáticas: relação com o ambiente, colheita e metabolismo secundário. Viçosa: UFV. 139p.

BHAN MK; KANTI R; KAK SN; PAL S; REKHA K. 1999. Response of new improved strain RL-931 of Cymbopogon to nitrogen fertilization. Journal of Medicinal and Aromatic Plant Sciences, 21: 1027-1029.

BRAGA R. 1976. Plantas do nordeste, especialmente do Ceará. Fortaleza: UFC. 540p.

DHAR AK; DAHR RS; REKKA K; KOUL S. 1996. Effect of spacings and nitrogen levels on herb and oil yield, oil concentration and composition in three selections of Cymbopogon jwarancusa (Jones) Schultz. Journal of Spices and Aromatic Crops, 5: 120 126.

GUENTHER E. 1992. The essential oils: individual essential oils of the plant families. Malabar: Krieger Publication. 3894p.

KHODE PP; GHATOL PU; DHUMAL VM; MAHAKAL MM. 1999. Effect of spacing and nitrogen levels on foliage and oil yields of palmarosa grass (Cymbopogon martinii). Agricultural Science Digest of Karnal, 19: 264-266.

KIEHL EJ. 1985. Fertilizantes orgânicos. Piracicaba: Agronômica Ceres. 492p.

LEAL TCAB; FREITAS SP; SILVA JF; CARVALHO AJC. 2001. Avaliação do efeito da variação estacional e horário de colheita sobre o teor foliar de óleo essencial de capim-cidreira (Cymbopogon citratus (DC) Stapf). Revista Ceres, 48: 445-453.

LORENZI H; MATOS FJA. 2002. Plantas medicinais no Brasil: nativas e exóticas cultivadas. Nova Odessa: Plantarum. 544p.

MING LC. 1998. Adubação orgânica no cultivo de Lippia alba (Mill) N.E.Br. Verbenaceae. In MING LC; SCHEFFER MC; CORREA JR C; BARROS IBC; MARROS JK. Plantas medicinais, aromáticas e condimentares: avanços na pesquisa agronômica. Botucatu: UNESP. 1: 165-191.

MING LC; FIGUEIREDO RO; MACHADO SR; ANDRADE RMC. 1996. Yield of essential oil and citral content in different parts of lemon grass leaves (Cymbopogon citratus (D.C.) Stapf.) Poaceae. Acta Horticulturae 426: 555559.

OLIVEIRA VA; LUNKES JA; ARGENTA JA; OLIVEIRA JA; DIAS DR. 2002. Efeito da adubação mineral e orgânica sobre a produção de biomassa e óleo essencial de capimlimão (Cymbopogon citratus Stapf). Pro Homine, 1: 24-27.

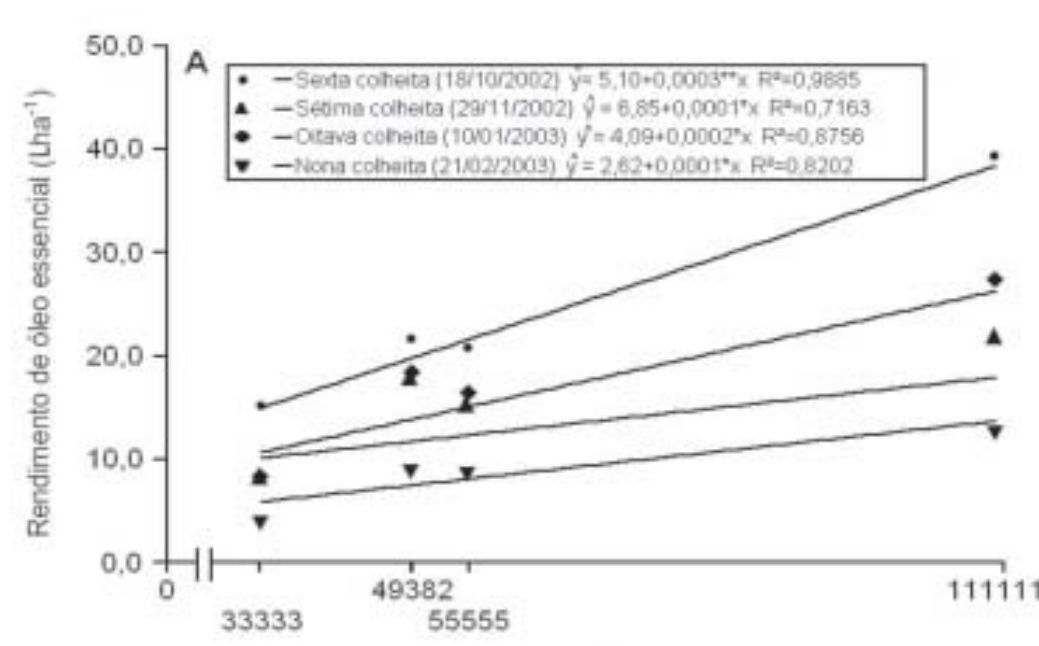

Densidade de plantio (plantas ha-1)

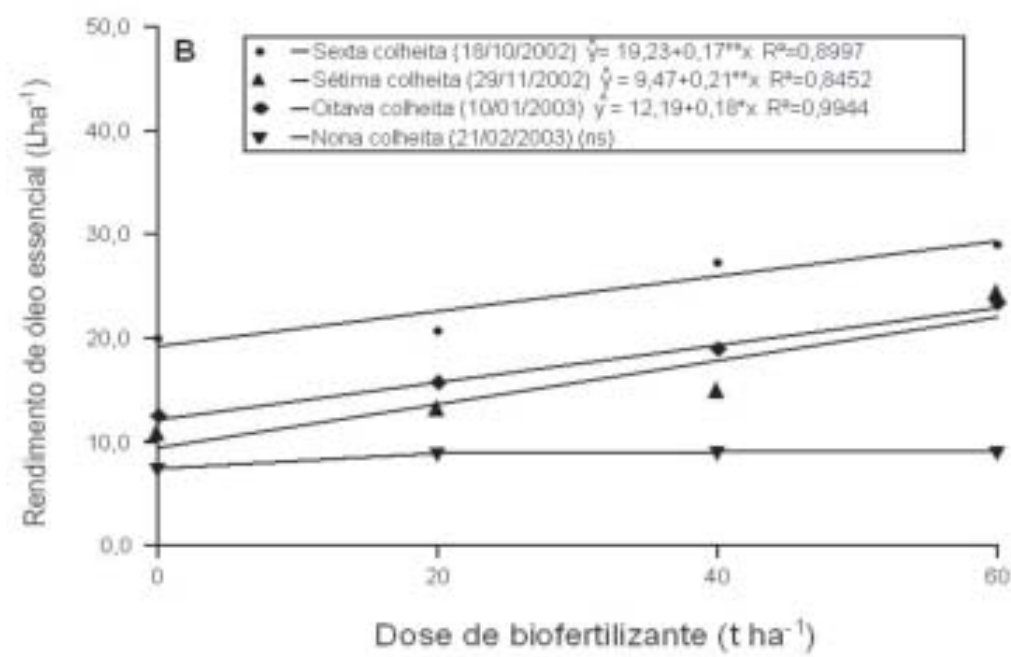

Figura 2. Rendimento médio (l ha $\left.{ }^{-1}\right)$ de óleo essencial de capim-limão entre a sexta e nona colheita, em função de diferentes densidades de plantio (A) e doses de biofertilizantes (B) (mean yield ( $\mathrm{ml} 100 \mathrm{~g}^{-1}$ ) of essential oil of lemon grass on the $6^{\text {th }}$ to $9^{\text {th }}$ harvest, depending on planting densities (A) and biofertilizer doses (B)). São Cristóvão-SE, UFS, 2003.

SILVA PA; BLANK AF; ARRIGONI-BLANK MF; BARRETO MCV. 2003. Efeitos da adubação orgânica e mineral na produção de biomassa e óleo essencial do capim-limão [Cymbopogon citratus (D.C.) Stapf]. Ciência Agronômica, 34: 92-96.

SINGH A; BALYAN SS; SHAHI AK. 1982 Cultivation of Jammu lemongrass in North India under irrigated conditions. In: ATAL, CK; KAPUR, BM (eds). Cultivation and utilization of aromatic plants. Jammu-Tawi, India: Council of Scientific and Industria Research. p. 325-330.
YADAV RL; MOHAM R; RAM M. 1983. Yield and quality of essential oil of japanese mint as affected by N-rates and row-spacing. Mandras Agriculture Journal, 70: 454-457. 